\title{
Distributed Interference Alignment in Cognitive Radio Networks
}

\author{
$\mathrm{Yi} \mathrm{Xu}$ and Shiwen Mao \\ Department of Electrical and Computer Engineering, Auburn University, Auburn, AL, USA
}

\begin{abstract}
In this paper, we investigate the problem of incorporating two advanced physical layer technologies, i.e., multipleinput and multiple-output (MIMO) and distributed interference alignment, in cognitive radio (CR) networks. We present a cooperative spectrum leasing scheme for primary and secondary users to trade off between data transmission and revenue collection/payment. A Stackelberg game is formulated, where the primary user is the leader and the secondary users are followers. With backward induction, we derive the unique Stackelberg Equilibrium, where no player can gain by unilaterally changing strategy, as well as the optimal strategies. We find spectrum leasing is always beneficial to enhance the utilities of primary and secondary users. The proposed scheme outperforms a nospectrum-leasing scheme and a cooperative scheme presented in the literature with considerable gains, which demonstrate the benefits of spectrum leasing and distributed interference alignment and validate the efficacy of the proposed scheme.
\end{abstract}

\section{INTRODUCTION}

Due to the tremendous increase in wireless data, radio spectrum is quickly depleted. However, according to the FCC report [1], while some licensed bands are overcrowded, many others are underutilized. Under traditional fixed spectrum allocation policy, when licensed users (or, primary users) are not active, the channels assigned to them are wasted (termed as spectrum opportunities). Cognitive radios (CR) are proposed as a new wireless paradigm for exploiting such spectrum opportunities, to enable flexible and efficient access to radio spectrum. In CR networks, unlicensed users (or, secondary users) can access the licensed band opportunistically, while primary users gain by collecting revenue for spectrum leasing.

Such a CR paradigm has been shown to have high potentials to enhance spectrum efficiency [2]. As significant advances are made in many aspects of CR research, such as spectrum sensing and dynamic spectrum access, it is also desirable to incorporate advanced physical layer techniques into CR networks. One of such techniques is Multiple-Input and MultipleOutput (MIMO), which can be used to reduce bit error rate, transmit more packets, or strengthen the signal to interference and noise ratio (SINR). In the past decade, MIMO has evolved from a theoretic concept to a technology that can be widely used in practice [3]. It is desirable to exploit MIMO for enhanced primary and secondary transmissions.

The second physical layer technology is interference alignment, a significant breakthrough that exploits interference in interference limited wireless networks [4]. Traditionally, if interference is small, it is simply treated as background noise; if interference is large, it can be decoded first and then removed from the received signal (i.e., interference cancellation); if interference is comparable to the desired signal, we usually try to avoid this case by orthogonalizing the channels or adopt a medium access control (MAC) scheme [5], [6]. Unlike traditional approaches, interference alignment casts interference to half of the received signal space to achieve a normalized Degree of Freedom (DoF) of $K / 2$, where $K$ is the number of interfering users. Since an interference-free channel only has a normalized DoF of 1 , substantial system throughput gain can be achieved with interference alignment when $K$ is large. For interference alignment, a strong requirement is the availability of global channel state information (CSI) at every node. To relax this requirement, distributed interference alignment is investigated and an iterative algorithm is proposed in [7] to achieve interference alignment with local CSI.

In this paper, we investigate how to incorporate these two advanced physical layer technologies, i.e., MIMO and distributed interference alignment, in CR networks. The CR network consists of a primary user and multiple secondary users, each with $N$ antennas. Time is divided into equal length time slots with a normalized length. The primary user has some data to send and requires a certain non-zero data rate in each time slot. It also leases spectrum to secondary users for more revenue. Secondary users pay the primary user for data transmission in the time slot. In the proposed cooperative spectrum leasing scheme, the primary user divides the time slot into three phases: (i) in Phase I, only the primary user transmits with MIMO; (ii) in Phase II, the primary user and a selected set of secondary users transmit simultaneously using distributed interference alignment; (iii) in Phase III, only selected secondary users transmit with distributed interference alignment. The primary user decides the division of the three phases, selects the set of secondary users for spectrum leasing, and collects a revenue from the selected secondary users proportional to their transmit powers (or, data rates).

We find such a cooperative spectrum leasing framework fits well with the Stackelberg game theory [8]. In the formulated Stackelberg game, the primary user is the leader and the secondary users are followers. The leader decides the division of a time slot into three phases and selection of followers, aiming to balance its own data transmission and revenue collection by leasing spectrum. Once the leader decisions are made, a follower can choose a transmit power (and the corresponding data rate) based on how much it is willing to pay. We define the Stackelberg Equilibrium where neither the primary user nor any secondary user could gain by unilateral change of strategy. 
We present a rigorous analysis with the backward induction method [8] and derive the unique Stackelberg Equilibrium for the cooperative spectrum leasing game.

We find the most desirable scenario for secondary users is to have only Phase III in the time slot with only 3 players. The strategy for the primary user depends on the number of secondary users. With more than $2 N-2$ secondary users, exactly $2 N-2$ secondary users will be selected, each having one interference free channel, and there will be only Phase II in the time slot. With fewer than $2 N-2$ secondary users, all secondary users will be selected and there will be only Phases II and III in the time slot. Therefore, spectrum leasing is always helpful for increasing the utilities of both the primary and secondary users. In the simulation study, we first compare the proposed scheme with a scheme without spectrum leasing to demonstrate the benefits of spectrum leasing. We then compare the proposed scheme with the cooperative scheme presented in [9] to demonstrate the efficacy of distributed interference alignment. Significant performance gains are achieved by the proposed scheme in these simulations.

The remainder of this paper is organized as follows. In Section II, we present the preliminaries and system model. We define the Stackelberg game in Section III and derive the unique Stackelberg equilibrium in Section IV. Simulation results are presented in Section V and related work is reviewed in Section VI. Section VII concludes this paper.

\section{Preliminaries And System Model}

\section{A. MIMO and Distributed Interference Alignment}

This paper is closely related to MIMO and distributed interference alignment. We brief review the preliminaries in this section. More details can be found in [3], [7].

1) MIMO Capacity Basics: With the advance of antenna technology, it is now feasible to equip wireless devices with multiple antennas. In general, three types of performance gains can be achieved with MIMO, namely, diversity gain, multiplexing gain, and antenna gain. In this paper, we focus on multiplexing gain, namely, DoF. We assume that all transmitters and receivers have the same number of antennas.

For a MIMO system with $N \geq 2$ antennas, assume that the CSI $\mathbf{H}$ is known at the transmitter. Since the MIMO channel can be decomposed into $d$ parallel channels, the channel capacity is given by [10]

$$
C=\max _{p_{i}: \sum_{i} p_{i} \leq P} \sum_{i=1}^{d} \log \left(1+\frac{\sigma_{i}^{2} p_{i}}{N_{0}}\right),
$$

where $P$ denotes the total transmitter power limit, $p_{i}$ is the power allocated to the $i$-th parallel channel, $\sigma_{i}^{2}=\lambda_{i}$ and $\lambda_{i}$ is the $i$-th largest eigenvalue of matrix $\mathbf{H H}^{H}$. Note that bandwidth is normalized throughout this paper.

In the high SNR region, equal power allocation is shown to be sub-optimal, but is easier for mathematical modeling than water-filling. When the transmit power is $P / d$ for each parallel

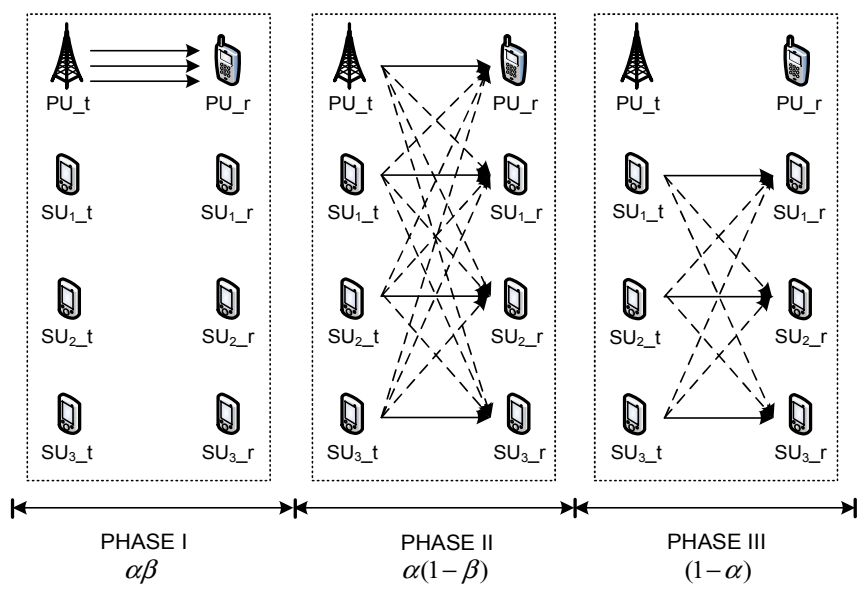

Fig. 1. The three-phase operation of the MIMO CR network with distributed interference alignment.

channel, the total capacity can be approximated as

$$
\begin{aligned}
C & \approx \sum_{i=1}^{d} \log \left(1+\frac{P \sigma_{i}^{2}}{d N_{0}}\right) \approx \sum_{i=1}^{d} \log \left(\frac{P \sigma_{i}^{2}}{d N_{0}}\right) \\
& =d \log (S N R)+\sum_{i=1}^{d} \log \left(\frac{\sigma_{i}^{2}}{d}\right) .
\end{aligned}
$$

The second item in (2) is negligible when the SNR is high. We thus ignore this term in the following sections.

2) Distributed Interference Alignment: The basic idea of interference alignment is to cast the interference to no more than half of the received signal space, and leave the other half clean and recognizable. If there are $K$ users, totally $K / 2$ normalized DoF could be achieved. The system throughput can be greatly enhanced when $K$ is large. For $K=0$ and 1 , there is no interference; for $K=2$, the normalized DoF is 1 , which is trivial. Therefore, we only consider the case where the number of interfering nodes $K$ satisfies $K \geq 3$. It is worth noting that, to align interference perfectly, global CSI is required at every participating node. To overcome this challenge, an iterative distributed interference alignment algorithm was proposed in [7], which only requires local CSI at each interfering node. In [11], a system is said to be proper if it satisfies $d \leq \frac{2 N}{K+1}$. We consider a proper system to be feasible for distributed interference alignment for simplicity.

\section{B. System Model and Assumptions}

The MIMO CR network is illustrated in Fig. 1. There are one primary user and $K_{T}$ secondary users sharing the licensed spectrum, each with $N$ antennas. We consider a time-slotted system, where each time slot is normalized to 1 unit in length and is divided into three phases, with lengths $\alpha \beta, \alpha(1-\beta)$, and $(1-\alpha)$, respectively, for fractions $0 \leq \alpha \leq 1$ and $0 \leq \beta \leq 1$.

In Phase I, the primary user transmits its packets at the highest rate using MIMO, and all secondary users remain silent. The DoF for the primary user is $d_{I}=N$. The achievable rate of the primary user in Phase $I$ is:

$$
R_{P}^{I}=d_{I} \log (S N R),
$$


where SNR is assumed to be constant during a time slot.

We assume that the primary user always has a finite amount of packets to send in each time slot. After a period of high data rate transmission (with length $\alpha \beta$ ), the primary user has the incentive to lease the spectrum to secondary users to increase its utility, by collecting revenue from selected secondary users (but at the cost of a lower data rate for itself). In Phase II, the primary user and $K \in\left[0, K_{T}\right]$ selected secondary users transmit simultaneously using distributed interference alignment, with a DoF of $d_{I I}=\left\lfloor\frac{2 N}{K+2}\right\rfloor$. A selected secondary user makes payments that is proportional to its transmit power (i.e., its data rate), and the primary user collects payments from all selected secondary users. The achievable rate of the primary user in Phase II is

$$
R_{P}^{I I}=d_{I I} \log (S N R) .
$$

The achievable rate of secondary user $S_{i}$ in Phase II is

$$
R_{S_{i}}^{I I}=d_{I I} \log \left(S N R_{i}\right),
$$

where $S N R_{i}=P_{i} / N_{0}$ is the $S N R$ for each selected secondary user, which is assumed to be constant in a time slot.

In Phase III, the primary user stops its transmission and leases the spectrum to selected secondary users, which transmit using distributed interference alignment with $d_{I I I}=\left\lfloor\frac{2 N}{K+1}\right\rfloor$. In Phase III, the achievable rate of secondary user $S_{i}$ is

$$
R_{S_{i}}^{I I I}=d_{I I I} \log \left(S N R_{i}\right) .
$$

We assume a common control channel for the primary user and secondary users to exchange precoding and interference cancellation matrices, the weight factor information, and the fractions $\alpha$ and $\beta$. Channel estimation is completed before data transmissions. Note that the DoFs are integers.

\section{StACKelberg GAME Formulation}

In the MIMO CR network, the primary user decides the division of a time slot into three phases and selection of secondary users, while balancing its own data transmission and revenue collection by leasing spectrum. Once the decisions are made by the primary user, a secondary user can choose a transmit power (and the corresponding data rate) based on how much it is willing to pay. Such interactions fit perfectly with the Stackelberg game model [8].

In this section, we formulate a Stackelberg game for the MIMO CR network with distributed interference alignment. The primary user is the leader and the secondary users are followers. The strategy of the primary user is given by

$$
S_{P}=\left\{\alpha, \beta, K \mid 0 \leq \alpha \leq 1,0 \leq \beta \leq 1,3 \leq K \leq K_{T}\right\} .
$$

The secondary user strategy is to find a transmit power $P_{i}$, as

$$
S_{S_{i}}=\left\{P_{i} \mid 0 \leq P_{i} \leq P_{\max }\right\}, \quad \forall i
$$

Here we assume that $P_{\max } \geq 2 w_{S} N / C_{0}$, where $C_{0}$ is unit price for secondary user transmit power (see (9)) and $w_{S}$ is the weight factor for secondary user utility (see (10)).
The primary user transmits its data in Phases I and II, and collects revenue in Phases II and III. The utility of the primary user is the sum of data transmitted and revenue collected, as

$$
U_{P}=w_{P} f_{P}\left(R_{P}\right)+\sum_{k=1}^{K} C_{0} P_{i}
$$

where $R_{P}=\alpha \beta R_{P}^{I}+\alpha(1-\beta) R_{P}^{I I}$ is the amount of primary user data transmitted, $w_{P}$ is a weight factor, $C_{0}$ is the unit price for secondary user power, and $f_{P}(x)$ is the satisfaction function of the primary user. Since the primary user always has some data to send, it requires a minimum data rate. Naturally we choose $f_{P}(x)=\ln (x), x \geq 0$. The negative value for very small $x$ serves as a penalty that forces the primary user to achieve a minimum data rate. From the shape of $f_{P}(x)$, the primary user is enthusiastic about data transmission at the beginning stage. After a period of high rate transmission, even a great increase in data transmission can only result in a small increase in its satisfaction. Since the primary user is rational and selfish, it aims to maximize $U_{P}$ by controlling the lengths of the three phases and selecting secondary users to participate in the game. By adjusting weight $w_{P}$, the primary users can trade off between data transmission and revenue collection.

Selected secondary users transmit their data during Phases II and III and make a one-time payment to the primary user. The utility of a secondary user $S_{i}$ is given by

$$
U_{S_{i}}=w_{S} f_{S}\left(R_{S_{i}}\right)-C_{0} P_{i} .
$$

where $R_{S_{i}}=\alpha(1-\beta) R_{S_{i}}^{I I}+(1-\alpha) R_{S_{i}}^{I I I}$ is the amount of data $S_{i}$ transmits, $f_{S}(x)$ is the secondary user satisfaction function, and $w_{S}$ is the weight factor. To simplify notation, we assume identical $w_{S}$ for all secondary users. Since the essence of CR is to opportunistically exploit spectrum, we choose $f_{S}(x)=x$, indicating that the secondary users operate in the best-effort manner. The weight $w_{S}$ allows a secondary user to trade off between data transmission and payment.

Therefore we define a Stackelberg game, with players, their roles, strategies ((7) and (8)), and utilities ((9) and (10)) specified. We provide a thorough analysis of the game with respect to the existence and uniqueness of the Stackelberg Equilibrium and optimal strategies in Section IV.

\section{Performance Analysis and Solution Strategy}

Let $\vec{P}^{*}$ be the vector of secondary user powers and $\vec{P}_{-i}^{*}=$ $\vec{P}^{*} \backslash P_{i}$. We first define Stackelberg Equilibrium as follows.

Definition 1. (Stackelberg Equilibrium) A strategy set $\left\{\alpha^{*}\right.$, $\left.\beta^{*}, K^{*}, \vec{P}^{*}\right\}$ is a Stackelberg Equilibrium of the game defined in Section III if the following conditions are satisfied:

1) $U_{P}\left(\alpha^{*}, \beta^{*}, K^{*}, \vec{P}^{*}\right) \geq U_{P}\left(\alpha, \beta, K, \vec{P}^{*}\right)$, for all $\alpha \in$ $[0,1], \beta \in[0,1]$, and $K \in\left[0, K_{T}\right]$.

2) $U_{S_{i}}\left(P_{i}^{*}, \vec{P}_{-i}^{*}, \alpha^{*}, \beta^{*}, K^{*}\right) \geq U_{S_{i}}\left(P_{i}, \vec{P}_{-i}^{*}, \alpha^{*}, \beta^{*}, K^{*}\right)$, for all $\alpha \in[0,1], \beta \in[0,1], K \in\left[0, K_{T}\right]$ and $i \in[1, K]$.

Using the backward induction method [8], we prove the uniqueness of the Stackelberg Equilibrium, and derive the unique Stackelberg Equilibrium (and the optimal strategy) for the game defined in Section III in the remainder of this section. 


\section{A. Secondary User Utility Maximization}

From (10), the utility of secondary user $S_{i}$ is given by $U_{S_{i}}\left(P_{i}\right)=w_{S}\left[\alpha(1-\beta) d_{I I} \log \left(P_{i} / N_{0}\right)+(1-\right.$ a) $\left.d_{I I I} \log \left(P_{i} / N_{0}\right)\right]-C_{0} P_{i}$. To maximize its utility, the secondary user solves the following maximization problem.

$$
\max _{0 \leq P_{i} \leq P_{\max }} U_{S_{i}}\left(P_{i}\right) .
$$

For given $\alpha$ and $\beta, U_{S_{i}}\left(P_{i}\right)$ is a concave function of $P_{i}$. Setting $d U_{S_{i}} / d P_{i}=0$, we derive the unique maximizer of (11), as

$$
P_{i}^{*}=\left[w_{S} \alpha(1-\beta) d_{I I}+w_{S}(1-\alpha) d_{I I I}\right] / C_{0} .
$$

Since $0 \leq \alpha \leq 1,0 \leq \beta \leq 1$ and $d_{I I} \leq d_{I I I} \leq 2 N$, we have

$$
P_{i}^{*} \leq w_{S} d_{I I I} / C_{0} \leq 2 w_{S} N / C_{0} \leq P_{\max }
$$

indicating that $P_{i}^{*}$ given in (12) is a feasible solution. It follows that the maximum utility of the secondary user is

$$
U_{S_{i}}^{*}=Y \log \left(Y /\left[2 C_{0} N_{0}\right]\right), i \in[1, K],
$$

where $Y=w_{S}\left[\alpha\left(d_{I I}-d_{I I I}\right)-\alpha \beta d_{I I}+d_{I I I}\right]$.

Since $U_{S_{i}}^{*}$ is a monotone increasing function of $Y$, and $d_{I I} \leq d_{I I I}$, it can be verified that $U_{S_{i}}^{*}$ is a monotone decreasing function of $\alpha$ and $\beta$. Since $\alpha(1-\beta) \geq 0$ and $(1-\alpha) \geq 0, U_{S_{i}}^{*}$ is a monotone increasing function of $d_{I I}$ and $d_{I I I}$. From a secondary user's perspective, the best scenario is $\alpha=0, \beta=0$, and $K=3$, i.e., the entire time slot is Phase III with the minimum number of followers. The selected secondary users enjoy the highest data rate. The primary user can only collect revenue from the three secondary users.

\section{B. Primary User Utility Maximization}

Given the optimal strategies of all the secondary users, we substitute $f_{P}\left(R_{P}\right)$ and $P_{i}^{*}$ into (9). It follows that

$$
\begin{aligned}
U_{P}(\alpha, \beta, K)= & w_{P} \ln \left[\alpha \beta R_{P}^{I}+\alpha(1-\beta) R_{P}^{I I}\right]+ \\
& K w_{S}\left[\alpha(1-\beta) d_{I I}+(1-\alpha) d_{I I I}\right] .
\end{aligned}
$$

The primary user solves the following problem to maximize its utility.

$$
\max _{0 \leq \alpha \leq 1,0 \leq \beta \leq 1,3 \leq K \leq K_{T}} U_{P}\left(\alpha, \beta, K, \vec{P}^{*}\right) .
$$

Maximization of the primary user utility is more complicated. We examine the problem for different parameter ranges and derive the local maximizer in each range. The global optimum is found by comparing the local maximizers. Without loss of generality, we assume $w_{P}=w_{S}$. The analysis can be easily extended to the case when $w_{P} \neq w_{S}$.

1) Case I When $K_{T} \geq(2 N-1)$ : Due to lack of space, we give the following Lemma without proving it.

Lemma 1. For $K_{T} \geq 2 N-1, U_{P}$ achieves its maximum when $\alpha=1, \beta=0, K=2 N-2$, and the maximum value is given by

$U_{P}^{* 1}(1,0,2 N-2)=w_{P} \ln (\log (S N R))+w_{S}(2 N-2)$.
2) Case II When $K_{T}=(2 N-2)$ : It can be readily concluded that the conclusion given in Section IV-B1 still holds. So we have the following theorem.

Theorem 1. When $K_{T} \geq 2 N-2, U_{P}$ is maximized when $\alpha=1, \beta=0, K=2 N-2$, and the maximum of $U_{P}$ is given in (16).

Note that when $K=2 N-2, d_{I I}=1, d_{I I I}=1$. Theorem 1 indicates that, when there are plenty of secondary users, to maximize the primary user's utility, we should select $2 N-2$ out of them so that each of the selected secondary user can have exactly one interference free channel. Since $\alpha=1$ and $\beta=0$, there is no Phase I and Phase III. To maximize the primary user utility, there's no need for the primary user to use MIMO transmission alone. Transmitting data with distributed interference alignment while collecting revenue from spectrum leasing is the best strategy for the primary user.

3) Case III When $3 \leq K_{T} \leq(2 N-3)$ : In this section, we consider the case when $3 \leq K_{T} \leq 2 N-3$. Due to lack of space, we omit the analysis in this case, which is similar to the cases when $K_{T} \geq(2 N-1)$ and when $K_{T}=(2 N-2)$. We summarize the results in Case III in the following theorem.

Theorem 2. When $K_{T} \leq 2 N-3, U_{P}$ is maximized when $\alpha=\frac{\left(K_{T}+1\right)\left(K_{T}+2\right)}{2 N K_{T}}, \beta=0, K=K_{T}$, and the maximum of $U_{P}$ is given by (17).

$$
\begin{aligned}
& U_{P}^{* 2}\left(\frac{\left(K_{T}+1\right)\left(K_{T}+2\right)}{2 N K_{T}}, 0\right) \\
= & w_{P} \ln \left[\log (S N R) \frac{K_{T}+1}{K_{T}}\right]+K_{T} w_{S}\left(\frac{2 N}{K_{T}+1}-\frac{1}{K_{T}}\right) .
\end{aligned}
$$

\section{The Unique Stackelberg Equilibrium}

We now summarize the analysis in Sections IV-A and IV-B. The unique Stackelberg Equilibrium of the game defined in Section III is given in the following theorem.

Theorem 3. The unique Stackelberg Equilibrium is given by:

$$
\begin{aligned}
& \left(\alpha^{*}, \beta^{*}, K^{*}\right)= \\
& \begin{cases}(1,0,2 N-2), & \text { if } K_{T} \geq 2 N-2 \\
\left(\frac{\left(K_{T}+1\right)\left(K_{T}+2\right)}{2 N K_{T}}, 0, K_{T}\right), & \text { if } 3 \leq K_{T} \leq 2 N-3\end{cases} \\
& P_{i}^{*}=\left[w_{S} \alpha^{*}\left(1-\beta^{*}\right) d_{I I}+w_{S}\left(1-\alpha^{*}\right) d_{I I I}\right] / C_{0}, \forall i .
\end{aligned}
$$

Since we can rewrite (19) as $P_{i}^{*}=w_{S}\left[\alpha\left(d_{I I}-d_{I I I}\right)-\right.$ $\left.\alpha \beta d_{I I}+d_{I I I}\right] / C_{0}$ and $d_{I I} \geq d_{I I I}, P_{i}^{*}$ is a monotone decreasing function of $\alpha$ and $\beta$. On the other hand, $P_{i}^{*}$ is a monotone increasing function of $d_{I I}$ and $d_{I I I}$, indicating that $P_{i}^{*}$ is a monotone decreasing function of $K$. The secondary users will adjust their transmitter power in light of $\alpha, \beta$ and $K$. The best scenario for them is $\alpha=0, \beta=0$ and $K=3$, for which there is only Phase III with the fewest players.

Knowing the optimal strategies of the secondary users, the primary user will set $\alpha=1, \beta=0$, and $K=2 N-2$ when there are a sufficient number of secondary users. Each selected secondary user has exactly one interference free channel, and there is only Phase II in the time slot. In this case, the primary 
user can collect as much revenue as possible while keeping a relatively low-rate data transmission. The secondary users' claim is satisfied in part. If there are not as many secondary users as needed, the primary user will set the parameters carefully according to (18). Under this condition, the primary user selects all the secondary users, discards Phase I, and makes a trade-off between Phase II and Phase III according to how many secondary users are there in the system.

\section{Simulation Study}

Simulations are conducted to validate the performance of the proposed scheme. We first compare the proposed scheme with a scheme without spectrum leasing to demonstrate the benefits of spectrum leasing. We then compare the proposed scheme with the cooperative scheme presented in [9] to demonstrate the efficacy of distributed interference alignment.

\section{A. With or Without Spectrum Leasing}

We first consider the case when there is a sufficient number of secondary users, i.e., $K_{T} \geq 2 N-2$, since in many realworld applications there are usually more secondary users than the number of antennas at each node. In Fig. 2, we plot the primary user utility $U_{P}^{*}$ versus the number of antennas $N$ and SNR. In the simulation, the weight factors are $w_{P}=w_{S}=$ 0.8 . The noise spectral density is $N_{0}=0.1$. Note that the maximum utility of the primary user without spectrum leasing can be shown to be:

$$
U_{P}^{* 3}=w_{P} \ln (N \log (S N R)) .
$$

It can be seen from Fig. 2 that there is a huge gap between the proposed scheme and the scheme without spectrum leasing. Note that the utility increase due to $S N R$ is less obvious than that due to $N$, since the impact of $S N R$ is diminished by the logarithms functions in (3) and (4). This clearly indicates that under the same setting, leasing spectrum to secondary users can greatly improve the primary user utility. Also it can be shown that, the utility of the proposed scheme is strictly larger than that of no spectrum leasing, for any feasible values of $w_{P}, N$ and SNR.

In Fig. 3, we examine the impact of weight $w_{P}$ on the primary user utility $U_{P}^{*}$. We plot the results with or without spectrum leasing, and for $N=2,4$, and 6 . It can be seen that when $w_{P}$ is increased, the gap between the proposed scheme and the scheme without spectrum leasing becomes larger. Although with increased $w_{P}$, the primary user emphasizes more on data transmission, the revenue is still increased at a higher speed with spectrum leasing. The gap also becomes larger when the number of antennas for each node is increased. This is also because the revenue increases faster with spectrum leasing than the no leasing scheme as $N$ is increased.

We then consider the case of an insufficient number of secondary users. In the simulation, there are $K_{T}=3$ secondary users. The number of antennas is $N=20$. We plot the primary user utility for the proposed scheme and the no-spectrumleasing scheme. We find that there's also a big gain achieved by the proposed scheme. This is consistent with our previous

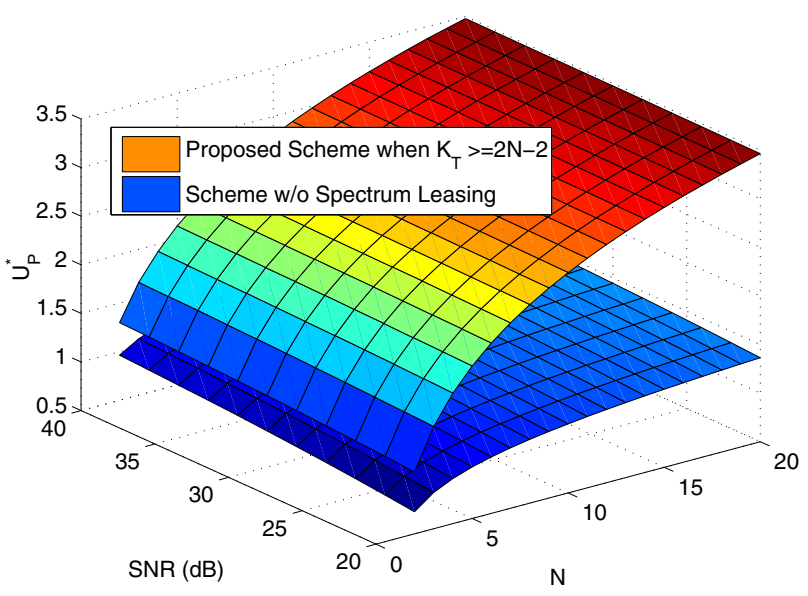

Fig. 2. Utility of the primary user in Log scale.

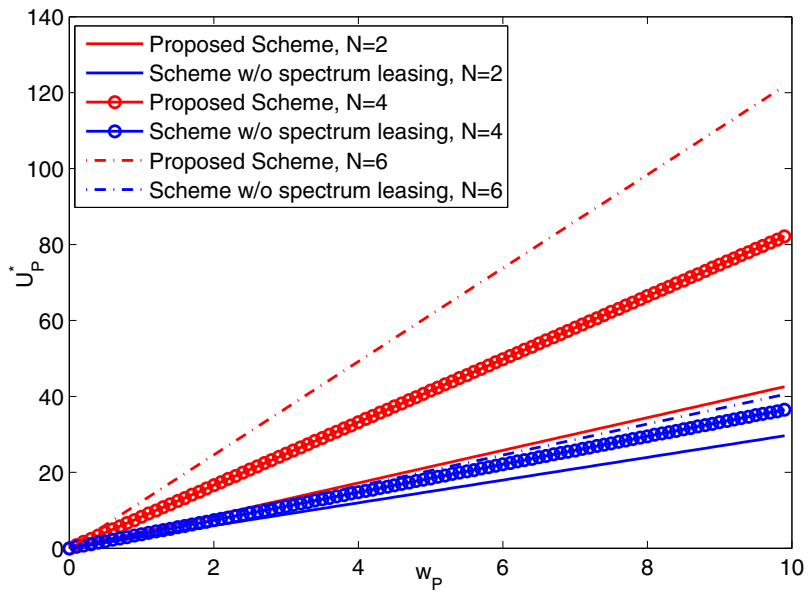

Fig. 3. Utility of the primary user when $K_{T} \geq 2 N-2$.

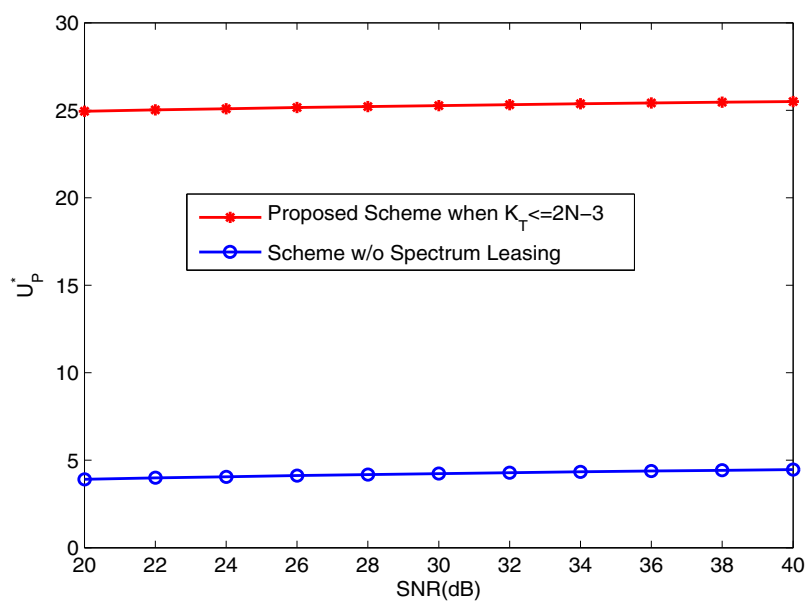

Fig. 4. Utility of the primary user when $K_{T} \leq 2 N-3$.

discussions. In this case, the primary user should still lease its spectrum to secondary users to maximize it own utility. 


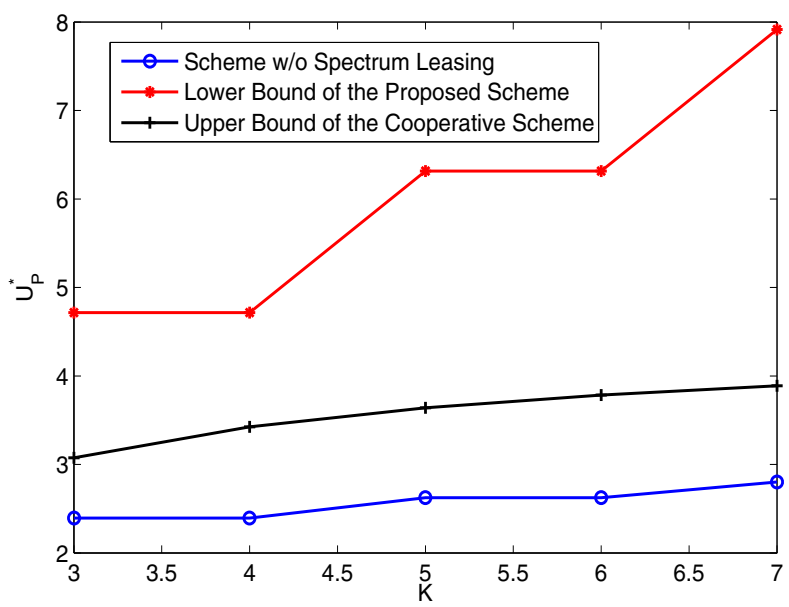

Fig. 5. Comparison of the proposed scheme with the cooperative scheme.

\section{B. With or Without Distributed Interference Alignment}

Finally, we compare the proposed scheme with the cooperative scheme presented in [9]. For a fair comparison, we replace the satisfaction function $f_{P}\left(R_{P}\right)=1 /\left[1+e^{-a\left(R_{P}-R_{0}\right)}\right]$ in [9] with $f_{P}\left(R_{P}\right)=\ln \left(R_{P}\right)$. We first derive an upper bound for the primary user utility in [9] (denoted as $U_{P}^{7}$ ) using our notation. Then we compare the proposed scheme with the derived upper bound. It follows that [9] where

$$
U_{P}^{7}=w_{P} \ln \left(R_{P}\right)+w_{S}(1-\alpha)(K-1) / \sum_{i}\left(1 / R_{S_{i}}\right),
$$

where

$$
\left\{\begin{array}{l}
R_{P}=\min \left\{\alpha \beta R_{P S}, \alpha(1-\beta) R_{S P}\right\} \\
R_{P S}=\log \left(1+\frac{\min _{i}\left|h_{P S, i}\right|^{2} P}{N_{0}}\right) \\
R_{S P}=\log \left(1+\frac{\left|h_{P}\right|^{2} P}{N_{0}}+\sum_{i} \frac{\min _{i}\left|h_{S P, i}\right|^{2} P}{N_{0}}\right) \\
R_{S_{i}}=\log \left(1+\frac{\left|h_{S_{i}}\right|^{2} P}{N_{0}}\right)
\end{array}\right.
$$

and the $h$ 's are channel states. Since

$$
\begin{aligned}
& R_{P}=\min \left\{\alpha \beta R_{P S}, \alpha(1-\beta) R_{S P}\right\} \\
& \leq \alpha \frac{R_{P S} R_{S P}}{R_{P S}+R_{S P}}<\alpha R_{P S} \\
& \leq \alpha \log \left(1+P / N_{0}\right), \text { and } \\
& R_{S_{i}}=\log \left(1+\left|h_{S_{i}}\right|^{2} P / N_{0}\right) \leq \log \left(1+P / N_{0}\right),
\end{aligned}
$$

we have:

$$
\begin{aligned}
U_{P}^{7}< & w_{P} \ln [\alpha \log (1+S N R)]+ \\
& w_{S}(1-\alpha)(1-1 / K) \log (1+S N R) .
\end{aligned}
$$

Define $f_{6}(\alpha)=\ln [\alpha \log (1+S N R)]+(1-\alpha)(1-1 / K) \log (1+$ $S N R)$. For $S N R \geq 3$ (true for the high SNR region), $f_{6}(\alpha)$ is maximized at $\hat{\alpha}=K /[(K-1) \log (1+S N R)]$. Substitute $\hat{\alpha}$ into (23), we have:

$$
U_{P}^{7}<w_{P} \ln \left(\frac{K}{K-1}\right)+w_{S}\left[\frac{K-1}{K} \log (1+S N R)-1\right],
$$

which provides an upper bound for the the utility of the cooperative scheme.
In Fig. 5, we plot the simulation results for the proposed scheme, the cooperative scheme, and the no-spectrum-leasing scheme. In the simulations, since the number of antennas must satisfy $\left\lfloor\frac{2 N}{K+2}\right\rfloor \geq 1$, as the number of $K$ varies, we set $N=\left\lceil\frac{K+2}{2}\right\rceil$. So we are actually comparing the lower bound of our proposed scheme with the upper bound of the cooperative scheme. It can be seen from Fig. 5 that both spectrum leasing schemes outperform the no-spectrum-leasing scheme. Furthermore, the proposed scheme outperforms the cooperative scheme with considerable gains. Such gains justify the efficacy of distributed interference alignment, which greatly enhance the overall system capacity.

\section{RELATED WORK}

This paper is closely related to the research on CR networks. For a general survey of $\mathrm{CRs}$, interested readers are referred to [2]. In a CR network, the primary user is either aware or unaware of the existence of the secondary users. This paper falls into the first category. The primary user is not only aware of the existence of the secondary user, but also knows the impact of the rules on the secondary user behavior. Most of the previous work, such as [9], [13]-[16], only considered the single antenna case, while we consider multiple antennas and exploit multiplexing gain in this paper.

This paper is also related to the research on interference alignment. In [4], the authors introduced the interference alignment technique. The significance of their work is that, by adopting interference alignment, the system is no longer interference limited. With symbol extension, the system could achieve a normalized DoF of $K / 2$. Another important issue, the feasibility condition, was investigated in [11] for structureless generic wireless channels. For wireless channels with a structure, such as diagonal channels, our recent paper [17] investigated the application of interference alignment in multi-user OFDM networks. To address the concern on the global CSI requirement, a distributed interference alignment algorithm was proposed in [7], which only requires local CSI. In [18], interference alignment and cancellation were integrated to achieve enhance the throughput of MIMO WiFi networks. In [19], Li et al. proposed a general algorithm for the multi-hop mesh networks. This work was motived by these interesting papers. However, many of the related work mainly focused on physical layer issues. This paper considers how to adopt distributed interference alignment in a MIMO CR network with a novel Stackelberg game based approach.

\section{CONCLUSIONS}

In this paper, proposed a three-phase cooperative spectrum leasing scheme with distributed interference alignment. The system was modeled as a Stackelberg game. With backward induction, we derived the unique Stackelberg equilibrium. Through rigorous analysis, we found the best strategies for the primary user and secondary users under a broad range of conditions and parameters, and discussed practical implications. We also found that leasing spectrum to secondary users is always helpful for enhancing the primary user utility. 
Simulation results demonstrated that the proposed scheme outperformed a no-spectrum-leasing scheme and a cooperative scheme from prior work.

\section{ACKNOWLEDGMENT}

This work is supported in part by the US National Science Foundation (NSF) under Grants CNS-0953513 and CNS1247955, and through the NSF Broadband Wireless Access \& Applications Center (BWAC) at Auburn University.

\section{REFERENCES}

[1] Federal Communications Commission, "Spectrum Policy Task Force," Rep. ET Docket no. 02-135, Nov. 2002.

[2] A. Goldsmith, S. A. Jafar, I. Maric, and S. Srinivasa, "Breaking spectrum gridlock with cognitive radios: An information theoretic perspective," Proc. of The IEEE, vol. 97, no. 5, pp. 894-914, May 2009.

[3] J. Mietzner, R. Schober, L. Lampe, W. H. Gerstacker, and P. A. Hoeher, "Multiple-antenna techniques for wireless communications - A comprehensive literature survey," IEEE Commun. Surveys Tuts., vol. 11, no. 2, pp. 87-105, Second Quarter 2009.

[4] V. R. Cadambe and S. A. Jafar, "Interference alignment and degrees of freedom of the k-user interference channel," IEEE Trans. Inf. Theory, vol. 54, no. 8, pp. 3425-3441, Aug. 2008.

[5] Z. Shi, Stochastic Modeling, Correlation, Competition, and Cooperation in a CSMA Wireless Network. ProQuest, UMI Dissertation Publishing, 2011.

[6] Z. Shi, C. Beard, and K. Mitchell, "Analytical models for understanding space, backoff and flow correlation in CSMA wireless networks," Springer Wireless Networks, vol.19, no.3, pp.393-409, Apr. 2013.

[7] K. Gomadam, T. Gou, V. R. Cadambe, and S. A. Jafar, "A distributed numerical approach to interference alignment and applications to wireless interference networks," IEEE Trans. Inf. Theory, vol. 57, no. 6, pp. 3309-3322, June 2011.

[8] D. Fudenberg and J. Tirole, Game Theory. Cambridge, MA: MIT Press, 1993.

[9] J. Zhang and Q. Zhang, "Stackelberg game for utility-based cooperative cognitive radio networks," in Proc. ACM MobiHoc'11, New Orleans, LA, May 2011, pp. 23-31.

[10] D. Tse and P. Viswanath, Fundamentals of Wireless Communication. Cambridge, UK: Cambridge University Press, 2005.

[11] C. M. Yetis, T. Gou, S. A. Jafar, and A. H. Kayran, "On feasibility of interference alignment in mimo interference networks," IEEE Trans. Signal Process., vol. 58, no. 9, pp. 4771-4782, 2010.

[12] S. Boyd and L. Vandenberghe, Convex Optimization. Cambridge, UK: Cambridge University Press, 2004.

[13] A. A. Daoud, T. Alpcan, S. Agarwal, and M. Alanyali, "A Stackelberg game for pricing uplink power in wide-band cognitive radio networks," in Proc. IEEE CDC'08, Cancun, Mexico, Dec. 2008, pp. 1422-1427.

[14] Y. Xiao, G. Bi, and D. Niyato, "Distributed optimization for cognitive radio networks using stackelberg game," in Proc. IEEE ICCS'10, Singapore, Nov. 2010, pp. 77-81.

[15] D. Niyato, E. Hossain, and Z. Han, "Dynamics of multiple-seller and multiple buyer spectrum trading in cognitive radio networks: A game theoretic modeling approach," IEEE Trans. Mobile Comput., vol. 8, no. 8, pp. 1009-1022, Aug. 2009.

[16] D. Hu and S. Mao, "Cooperative relay with interference alignment for video over cognitive radio networks," in Proc. IEEE INFOCOM'12, Orlando, FL, Mar. 2012, pp. 2014-2022.

[17] Y. Xu and S. Mao, "On interference alignment in multi-user OFDM systems," in Proc. IEEE GLOBECOM 2012, Anaheim, CA, Dec. 2012

[18] S. Gollakota, S. D. Perli, and D. Katabi, "Interference alignment and cancellation," in Proc. ACM SIGCOMM'09, Barcelona, Spain, Aug. 2009, pp. 159-170.

[19] L. E. Li, R. Alimi, D. Shen, H. Viswanathan, and Y. R. Yang, "A general algorithm for interference alignment and cancellation in wireless networks," in Proc. IEEE INFOCOM'10, San Diego, CA, Mar. 2010. 